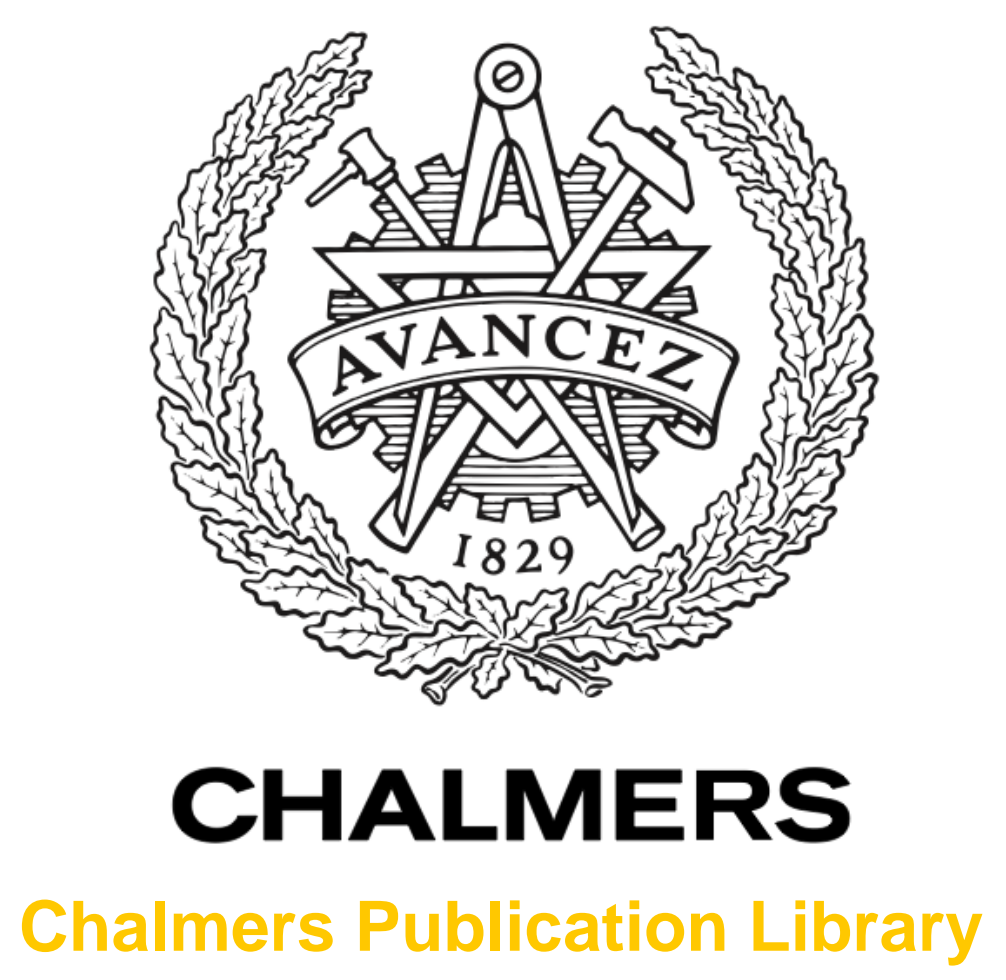

\title{
Uncertainty modelling in multi-criteria analysis of water safety measures
}

This document has been downloaded from Chalmers Publication Library (CPL). It is the author's version of a work that was accepted for publication in:

\section{Environment Systems and Decisions (ISSN: 2194-5403)}

Citation for the published paper:

Lindhe, A. ; Rosén, L. ; Røstum, J. (2013) "Uncertainty modelling in multi-criteria analysis of water safety measures". Environment Systems and Decisions, vol. 33(2), pp. 195-208.

http://dx.doi.org/10.1007/s10669-013-9442-9

Downloaded from: http://publications.lib.chalmers.se/publication/177692

Notice: Changes introduced as a result of publishing processes such as copy-editing and formatting may not be reflected in this document. For a definitive version of this work, please refer to the published source. Please note that access to the published version might require a subscription. 


\title{
Uncertainty modelling in multi-criteria analysis of water safety measures
}

\author{
Andreas Lindhe ${ }^{\mathrm{a}, *}$, Lars Rosén ${ }^{\mathrm{a}}$, Tommy Norberg ${ }^{\mathrm{b}}$, Jon Røstum ${ }^{\mathrm{c}}$ and Thomas J.R. Pettersson ${ }^{\mathrm{a}}$ \\ a Department of Civil and Environmental Engineering, Chalmers University of Technology, \\ SE-412 96 Gothenburg, SWEDEN. E-mail: andreas.lindhe@chalmers.se; Tel: +46 31772 2060; \\ Fax: +46317722107 \\ ${ }^{\mathrm{b}}$ Department of Mathematical Sciences, University of Gothenburg and Chalmers University of Technology, \\ SE-412 96 Gothenburg, SWEDEN. E-mail: tommy.norberg@chalmers.se \\ c SINTEF Building and infrastructure, Klæbuvegen 153, NO-7465 Trondheim, NORWAY. E-mail: \\ jon.rostum@sintef.no \\ d Department of Civil and Environmental Engineering, Chalmers University of Technology, \\ SE-412 96 Gothenburg, SWEDEN. E-mail: lars.rosen@chalmers.se \\ e Department of Civil and Environmental Engineering, Chalmers University of Technology, \\ SE-41296 Gothenburg, SWEDEN. E-mail: thomas.pettersson@chalmers.se \\ * Corresponding author
}

\begin{abstract}
Water utilities must assess risks and make decisions on safety measures in order to obtain a safe and sustainable drinking water supply. The World Health Organization emphasises preparation of Water Safety Plans, in which risk ranking by means of risk matrices with discretised probability and consequence scales is commonly used. Risk ranking enables prioritisation of risks but there is currently no common and structured way of performing uncertainty analysis and using risk ranking for evaluating and comparing water safety measures. To enable a proper prioritisation of safety measures and an efficient use of available resources for risk reduction, two alternative models linking risk ranking and multicriteria decision analysis (MCDA) are presented and evaluated. The two models specifically enable uncertainty modelling in MCDA and they differ in terms of how uncertainties in risk levels are considered. The need of formal handling of risk and uncertainty in MCDA is emphasised in the literature and the suggested models provide innovations that are not dependent on the application domain. In the case study application presented here, possible safety measures are evaluated based on the benefit of estimated risk reduction, the cost of implementation and the probability of not achieving an acceptable risk level. Additional criteria such as environmental impact and consumer trust may also be included when applying the models. The case study shows how safety measures can be ranked based on preference scores or cost-effectiveness and how measures not reducing the risk enough can be identified and disqualified. Furthermore, the probability of each safety measure being ranked highest can be calculated. The two models provide a stepwise procedure for prioritising safety measures and enable a formalised handling of uncertainties in input data and results.
\end{abstract}

Keywords: multi-criteria decision analysis (MCDA), uncertainty analysis, risk ranking, drinking water, risk reduction, water safety plan. 


\section{INTRODUCTION}

The overall goal within the drinking water sector is to provide the customers with an uninterrupted supply of water with high aesthetic quality and with a minimal risk to human health. However, because of potential hazards in the raw water sources, the treatment plants and the distribution systems, water supply systems and consumers are exposed to a wide range of risks. In addition, climate changes, societal development and emergence of new contaminants constantly provide new risks. As a result, water utilities continuously have to assess risks and prioritise water safety measures in order to obtain a safe and sustainable supply system.

Efficient risk management requires not only a proper identification and estimation of risks but also a thorough evaluation and comparison of safety measures, i.e. risk-reduction measures. Given the limited economic resources of every organisation, proper prioritisations of safety measures are necessary. Furthermore, in order to identify the best option it is necessary to consider several aspects, including uncertainties of estimates. The World Health Organization (WHO, 2011) emphasises the need of Water Safety Plans (WSPs) in which risks to the entire drinking water system, from source to tap, should be analysed and evaluated. There are currently few methods available for analysing risks to entire drinking water systems (Lindhe et al., 2009) and when performed, risk ranking methods with risk matrices are commonly used. With risk ranking we here refer to methods where undesired events are assessed based on discretised scales for the probability of occurrence and the negative consequences, and where the resulting risk is presented using one or several risk matrices. Risk ranking is suggested by, for example, Bartram et al. (2009) and Davison et al. (2005) as part of WSPs. By using risk ranking it is possible to identify the most severe risks but there is currently no common and structured way of using risk ranking to evaluate and compare water safety measures. Furthermore, risk ranking typically does not enable proper uncertainty analysis. Hence, there is a need of combining risk ranking and decision analysis and enabling structured uncertainty analysis.

Decision analysis of safety measures will always be associated with uncertainties related to, for example, initial risk levels, risk reduction and the cost of implementing measures. A primary objective of this work was therefore to include formal evaluation of uncertainties in decision analysis. Two decision models were developed with alternative approaches for uncertainty assessments to enable a further use of risk ranking to evaluate and compare possible safety measures. An approach based on multi-criteria analysis is used in the two models and as far as we know, no such model for application by water utilities has yet been described in the literature. The two models are presented, applied and evaluated to show advantages and limitations.

Water safety measures typically have several effects including, for example, a reduced health risk and a reduced number of supply interruptions. A decision analysis thus needs to consider several aspects to treat the decision problem in a proper manner and to provide useful results. Multi-criteria decision analysis (MCDA) is a generic term including different techniques for evaluating and comparing the performance of alternative options based on a set of criteria (Communities and Local Government, 2009; Keeney and Raiffa, 1993; Roy, 2005). The key criteria used in the application presented in this paper were risk reduction and cost, but additional criteria can be added according to the preferences of the decision-makers.

The need of the developed models was identified based on problems related to the drinking water sector. However, the models provide approaches and techniques generally applicable within the field of MCDA. The primary aims of this paper were: (1) to present two MCDA models based on risk ranking with alternative approaches for uncertainty analysis that can be 
practically applied by water utilities; (2) to evaluate the alternative models with respect to their theoretical and practical functionality; and (3) to exemplify model applications in a case study in Bergen, Norway.

\section{MCDA IN DRINKING WATER APPLICATIONS}

There is a large number of MCDA applications related to drinking water systems (e.g. Bouchard et al., 2010; Joerin et al., 2009). Hajkowicz and Collins (2007) conclude, in their review paper on MCDA for water resource management, that MCDA is used for water policy evaluation, strategic planning and infrastructure selection. They further identified challenges for water resource MCDA research. Two of the main conclusions were that there is a need for improved handling of risk and uncertainty in MCDA models, and a need of means for incorporating risk preferences of decision-makers.

Furthermore, Stewart (2005) emphasises that risk and uncertainty typically are critical issues in strategic planning problems and there is a need of more formal handling of these issues in MCDA. Thus, it can be concluded that there is a general need of additional techniques for handling and modelling uncertainties in MCDA. Hyde et al. (2004) show how uncertainties in most input data can be described using probability distributions and how the results can be calculated using Monte Carlo simulations. We have used a similar, but simplified, approach to uncertainty modelling.

To be able to evaluate water safety measures in a proper manner, the following aspects were identified as important to consider when developing the models presented here:

- An undesired event may cause different consequences (e.g. related to water quality or supply interruptions) and thus also different risks.

- A safety measure may affect several undesired events and for each event different risk types.

- A safety measure is intended to reduce risks but can also increase other risks.

- All risk types do not have to be considered equally important.

- When evaluating safety measure the cost of implementing them is an important aspect.

- In addition to risk reduction and cost, other criteria such as environmental effects should be possible to consider in a decision analysis.

- To fully understand a decision problem it is important to consider uncertainties.

\section{METHODS}

\subsection{Risk ranking}

The main steps to be performed when ranking risks and presenting the results using risk matrices are: (i) identify undesired events; (ii) define discretised probability and consequence scales, i.e. the axes in the risk matrix; (iii) define risk tolerability criteria, i.e. what risks (combinations of probability and consequence) are acceptable and what risks are unacceptable; (iv) assess the probability and the consequence of each event; and (v) plot the risks in the matrix and evaluate them based on their position. The probability and consequence scales are typically divided into four or five classes and can, for example, be referred to as low, medium, high and very high. The classes can also be assigned numerical values to enable calculations of risk priority numbers/risk scores (e.g. Figure 4 in Section 4.2). The latter type of risk ranking is often termed semi-quantitative but here all risk ranking is referred to as qualitative. Risk priority numbers are used in the models presented here, see 
Section 3.3. For further descriptions of risk ranking and risk matrices, see e.g. Bartram et al. (2009) and AS/NZS (2004).

Risk ranking using risk matrices is easy to apply and provides results that are easily communicated. However, the method also has limitations, see e.g. Burgman (2005) and Cox (2008). A major limitation is the inability to consider interactions of different events in a structured way. Other, quantitative and more complex, risk models based on logic tree approaches have been developed to overcome this weakness, see e.g. Lindhe et al. (2009; 2011) and Rosen et al. (2010). Risk ranking cannot replace more detailed quantitative risk assessment methods but it is commonly applied and useful results can be obtained if existing limitations are considered.

\subsection{Safety measures, events and risks}

The two MCDA models presented here were developed to evaluate safety measures aimed at reducing unacceptable risks. These measures are also referred to as risk-reduction measures. A measure may affect the probability and/or the consequence of one or several undesired events. Furthermore, the combination of the probability and the consequence of an event represents the risk caused by the event. An event may cause different types of consequences and thus also different risk types, such as water quality risks and risks related to supply interruptions. Hence, a safety measure may affect one or several events and for each event one or several risks may be affected. An undesired event can also be referred to as a hazardous event or just hazard. Here the term event is used.

\subsection{MCDA models}

The two MCDA models share the same basic approach and a key part is the estimation of the benefit that alternative risk-reduction measures may provide in terms of reduced risk levels. The risk reduction is estimated by analysing how each safety measure changes the probability and/or the consequence values, i.e. changes the position of the risks in the matrices. Furthermore, the costs of implementing the measures are considered to enable costeffectiveness and cost-benefit analysis. Since the number of criteria (benefit of risk reduction and cost) is limited in the models, it is simple type of MCDA. However, since additional criteria related to both positive and negative aspects of analysed safety measures easily can be added to the models, we refer to them as MCDA models.

The main differences between the two models are how uncertainties are considered and how the benefit of risk reduction is calculated. In the first model, discrete probability distributions are used and the model is therefore named the discrete model. The second model is named the beta model since Beta distributions are used to model uncertainties. A schematic description of the main steps in the MCDA models and their relation to risk ranking are presented in Figure 1. 


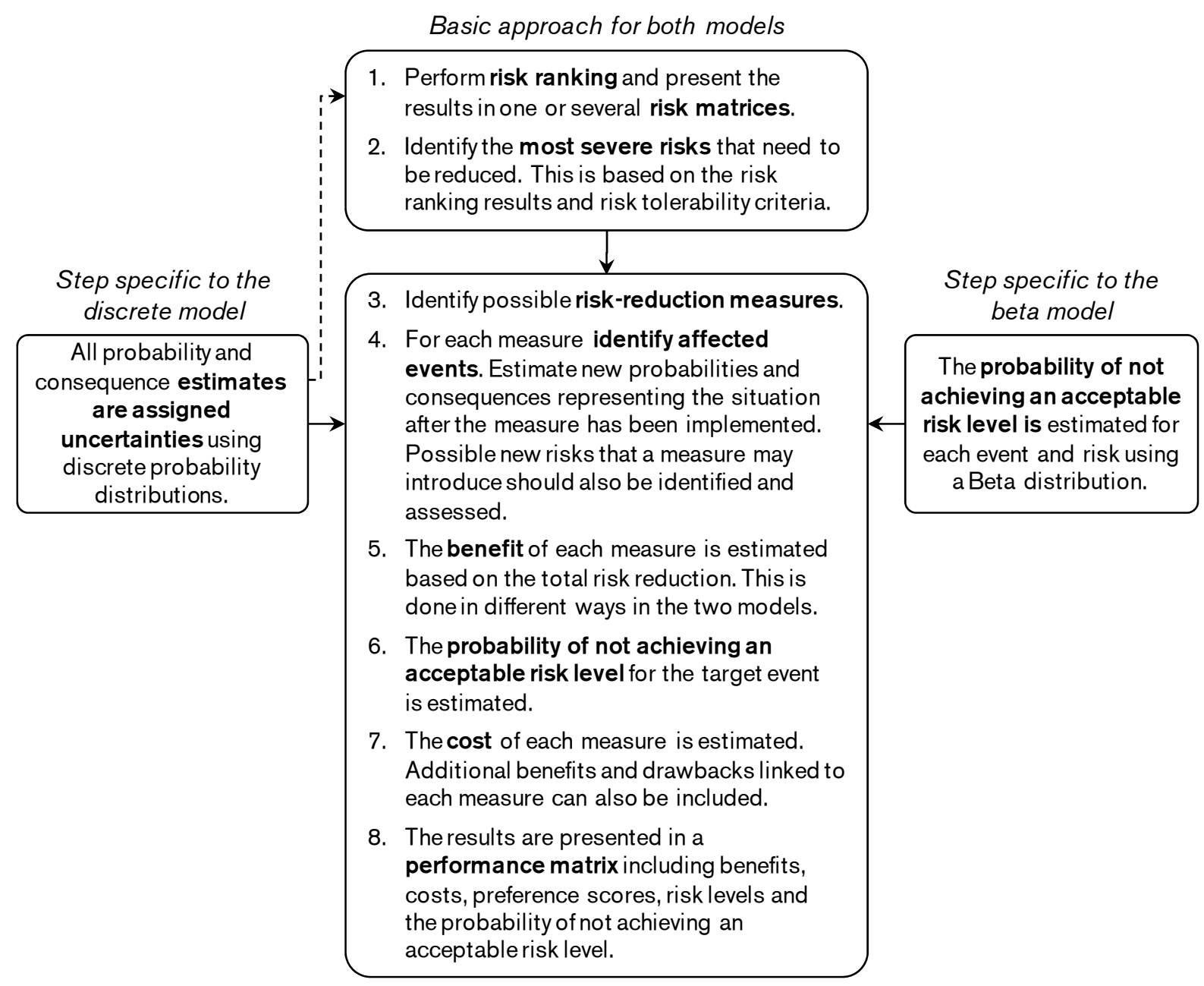

Figure 1 Schematic description of the two MCDA models. The steps common to the two models are presented in the middle and at each side the step specific to each model is presented.

\subsubsection{Basic approach}

A key component in the models presented here is to estimate the risk and more specifically the risk level changes (positive or negative) associated with a safety measure. Risk is commonly described as a combination of the probability and the consequence of an undesired event (AZ/NZS, 2004; IEC, 1995; Kaplan and Garrick, 1981; Kaplan et al., 2001). This approach is applied here and the risk $(R)$ caused by an event is

$$
R=f(p, c)
$$

where $p$ is the probability and $c$ the consequence of a specific undesired event. The probability and the consequence can be combined in different ways depending on how risk is expressed in the specific application.

The discretised scales in risk ranking can be ordinal, i.e. comparative and only expressed as high, medium, low, etc. Sometimes the classes are assigned values on an interval scale representing the severity in relation to the other classes. Furthermore, the probability and consequence values can be combined into a risk value expressed as a priority number. The scales may be assigned values in different ways and alternative ways of calculating the risk priority number exist. To include all relevant outcomes (i.e. very low as well as very high probability/consequence) the scales are often not linear but rather of a logarithmic nature. A possible and simple model for translating a set of classes $(x=1,2, \ldots, n)$ into values $\left(v_{x}\right)$ is 


$$
v_{x}=A^{x-1}
$$

where $A$ is a factor determining the difference between the value of the classes. For example, if a scale is divided into four classes and Equation (2) is used with $A=2$, then the classes are assigned values $1,2,4$ and 8 . When the risk is calculated and expressed as a risk priority number, different weights can be given to the probability and the consequence, representing the perception of the decision-maker. A possible equation for calculating the risk is

$$
R=p^{a} \cdot c^{b}
$$

where $a$ and $b$ are weights determining how much each parameter contributes to the risk.

Since an undesired event $(i)$ may cause more than one type of consequence we can rewrite Equation (1) as

$$
R_{i}=f\left(p_{i}, c_{i 1}, c_{i 2}, \ldots, c_{i n}\right)
$$

where $n$ different consequences are included. The probability of the event is considered to be independent of the consequences and therefore only one probability is included in Equation (4). Since there are $n$ consequences, the total risk caused by an event $\left(R_{i}\right)$ constitutes a set of $n$ risk levels $\left(R_{i k}, k=1,2, \ldots, n\right)$. The change a measure $(j)$ may achieve in risk type $k$ related to event $i\left(\Delta R_{j i k}\right)$ is

$$
\Delta R_{j i k}=R_{0 i k}-R_{j i k}
$$

where $R_{0 i k}$ is the initial risk level before any measure is taken and $R_{j i k}$ is the residual risk after measure $j(j>0)$ has been implemented. It is consequently the change in risk levels that is calculated. Note that a risk level change can be positive or negative.

\subsubsection{The discrete model}

In the discrete model the probability and the consequence values are assigned uncertainties and the benefit is calculated as a weighted sum of all reduced risk levels. A safety measure $(j)$ may affect a set of events $(i)$ and for each event a set of risks $(k)$ may be affected. The total benefit of a measure $\left(u_{j}\right)$ is

$$
u_{j}=\sum_{i} \sum_{k} \Delta R_{j i k} w_{k}
$$

where $\Delta R_{j i k}$ is the estimated risk reduction of measure $j$ related to event $i$ and risk $k$, and $w_{k} \geq 0$ are weighting factors determining how much the reduction of each risk type (e.g. quality risk or quantity risk) contributes to the total benefit.

Uncertainties in risk levels are considered by using discrete probability distributions to model uncertainties of the true probability and consequence values. For example, if a consequence is estimated to have a value of 2 (on an interval scale of 1,2, 4 and 8), this is assumed to be the most likely value but there is also a probability that the consequence is 1,4 or 8 . An example of how discrete probability distributions can be defined is presented in Table 1 . To facilitate model application it is suggested that a set of probability distributions are defined representing different levels of uncertainty about the probability and consequence values (Figure 2). The idea is that when the model is used, the most likely value (y-axis in Figure 2) is first estimated and afterwards a judgement is made regarding the level of uncertainty (x-axis in Figure 2) associated with this estimate. The set of distributions should be adjusted to fit the specific application and in addition to predefined distributions, it should be possible to define other distributions if no proper match is found. 
Table 1 Example of how discrete probability distributions for probability and consequence values can be defined. All distributions have a normalised entropy value of 0.67 .

\begin{tabular}{ccccc}
\hline \multirow{2}{*}{ Value } & \multicolumn{4}{c}{ Probability of each value } \\
\cline { 2 - 5 } & 1 & 2 & 4 & 8 \\
\hline 1 & 0.68 & 0.19 & 0.09 & 0.04 \\
\hline 2 & 0.12 & 0.70 & 0.12 & 0.06 \\
\hline 4 & 0.06 & 0.12 & 0.70 & 0.12 \\
\hline 8 & 0.04 & 0.09 & 0.19 & 0.68 \\
\hline
\end{tabular}

In Figure 2, all distributions in the same column represent the same degree of uncertainty but the most likely value differs. To be able to define distributions in such a consistent manner information about the entropy can be used (e.g. Box et al., 2005; Jaynes, 1968). Also other ways of expressing the uncertainty of the outcomes of discrete classes can be envisaged, see e.g. Rosén and Gustafson (1996) and Rosenbaum et al. (1997). The entropy is maximised by the uniform distribution. Hence, based on the number of classes in the discrete distribution $(n)$ the normalised entropy $H$ of the discrete random variable $V$, here the probability or consequence value $\left(v_{x}, x=1,2, \ldots, n\right)$, is

$$
H(V)=-(\ln n)^{-1} \sum_{x=1}^{n} p\left(v_{x}\right) \ln p\left(v_{x}\right)
$$

where $p\left(v_{x}\right) \geq 0$ is the probability of the value being $v_{x}$ and $\sum_{x=1}^{n} p\left(v_{x}\right)=1$. The normalised entropy can attain a value between 0 and 1 , and can be used to compare the uncertainty in distributions with different $n$. A value of 1 represents maximum uncertainty. The distributions in Table 1 all have a normalised entropy value of 0.67 and thus represents one column in Figure 2.

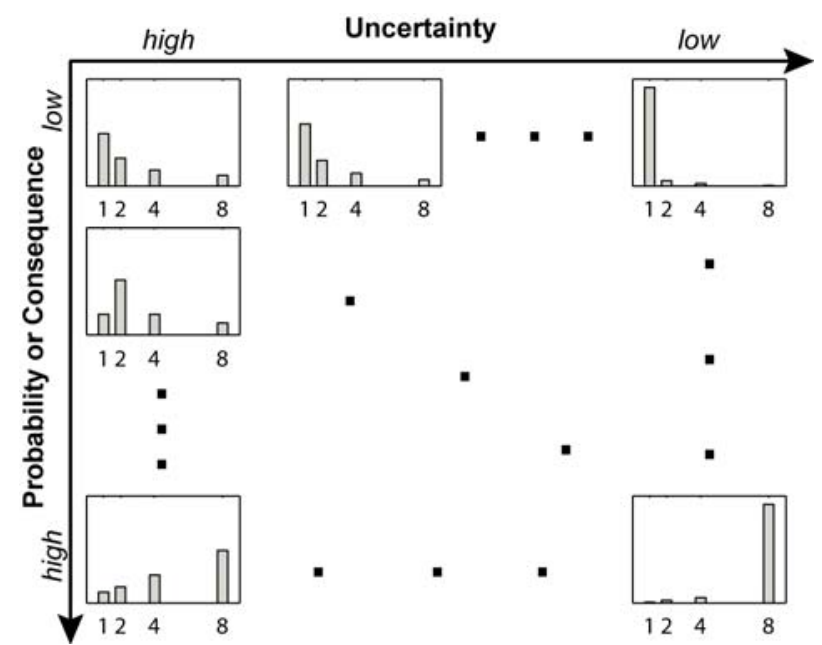

Figure 2 Example of how a set of discrete distributions can be defined and used as a guide when estimating the probability and consequence of undesired event.

By including uncertainties in the input data for the model, information on uncertainties in the calculated benefit of safety measures can be obtained. By using information about uncertainties it is possible to calculate the probability of not achieving an acceptable risk level. A safety measure may affect several events but the target event $(i=t)$ that the measure 
is designed to reduce is most important. Therefore, the probability of the risk associated with the target event $\left(R_{t k}\right)$ being higher than the acceptable/critical level of risk $k\left(R_{k}^{c}\right)$, i.e. $P\left(R_{t k}>R_{k}^{c}\right)$, is calculated.

\subsubsection{The beta model}

In the Beta model the uncertainties are not assessed for each probability and consequence value individually. Instead, the probability of achieving an acceptable risk level is directly estimated based on uncertainties about the risk level after a measure has been implemented, i.e. the final probability and consequence value. This probability is described using a Beta distribution which is flexible, can attain a wide variety of shapes and is often used for modelling uncertainties of proportions and probabilities, see e.g. Bedford and Cooke (2001). The probability of achieving an acceptable risk level is included in the calculation of the benefit since it is assumed that the greatest benefit is obtained when the risk is reduced to an acceptable level. Hence, a risk reduced from an unacceptable to an acceptable level is considered more beneficial compared to the same amount of risk reduction but where the final risk still is unacceptable. Furthermore, it is assumed that all risks may not be considered equally important, and that the risk reduction thus may be weighted. The total benefit of a safety measure $\left(u_{j}\right)$ that affects a set of events $(i)$ and for each event a set of risks $(k)$ is

$$
u_{j}=\sum_{i} \sum_{k} \Delta R_{j i k} l_{j i k} w_{k}
$$

where $\Delta R_{j i k}$ is the estiamted risk reduction of measure $j$ related to event $i$ and risk $k, l_{j i k}$ represents the probability of achieving an acceptable risk level and $w_{k} \geq 0$ are weighting factors determining how much the reduction of each risk type contributes to the total benefit. If there is an increase in the risk level, then $l_{j i k}$ is the probability of not having an acceptable risk. Consequently,

$$
l_{j i k}=\left\{\begin{array}{l}
l_{j i k}^{+} \text {if } \Delta R>0 \\
l_{j i k}^{-} \text {if } \Delta R<0
\end{array}\right.
$$

where $l_{j i k}^{+}$is the probability of having an acceptable risk level and $l_{j i k}^{-}$the probability of not having an acceptable risk level. In the remainder of the text we mainly refer to the case where the risk is reduced when calculating the benefit, since this is most common. The Beta distributions used to describe the probabilities $\left(l_{j i k}\right)$ are defined based on two criteria: (i) the most likely probability; and (ii) the uncertainties of this value. As in the discrete model, a set of distributions can be used to facilitate the selection of a proper probability distribution (Figure 3). It should of course be possible to define other distributions if no proper match is found. The Beta density function with shape parameters $\alpha$ and $\beta$ is defined as

$$
f(x)=\frac{\Gamma(\alpha+\beta)}{\Gamma(\alpha) \Gamma(\beta)} x^{\alpha-1}(1-x)^{\beta-1}
$$

where $\Gamma$ is the gamma function, $x \geq 0$ and $\alpha, \beta>0$. The shape parameters can, for example, be estimated based on the number of positive and negative observations, $\alpha-1$ and $\beta-1$ respectively. The sum of $\alpha$ and $\beta$ represents the uncertainty in the distribution, i.e. a high value indicates low uncertainty. 


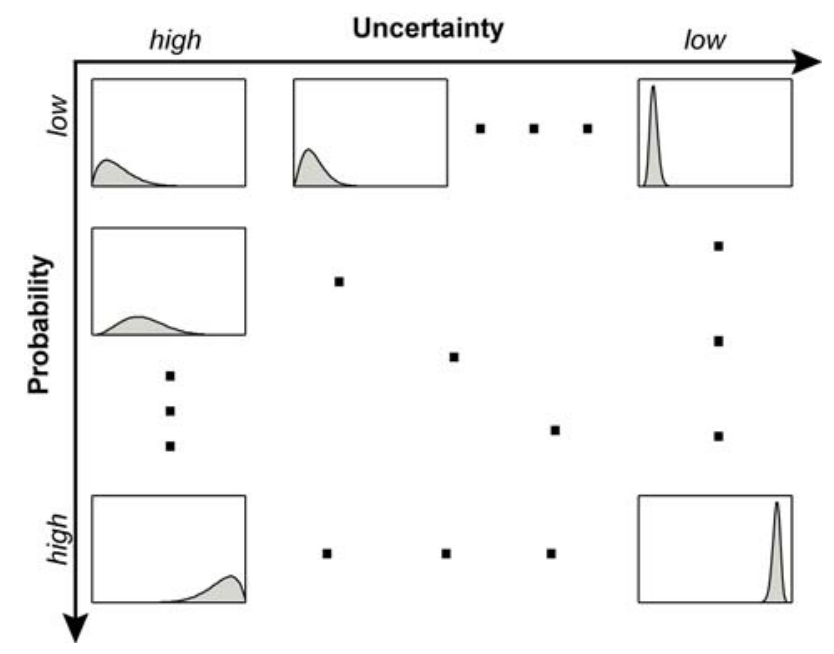

Figure 3 Example of how a set of Beta distributions can be defined and used as a guide when estimating the probability of achieving an acceptable risk level.

For the target event $(i=t)$ the probability $l_{j t k}^{-}$is equivalent to the probability of the risk $\left(R_{t k}\right)$ being higher than the acceptable risk $\left(R_{k}^{c}\right)$, i.e. $P\left(R_{t k}>R_{k}^{c}\right)$. As for the discrete model, this information is used in the final evaluation of the safety measures.

\subsubsection{Cost and other criteria}

In addition to the benefit of risk reduction also the cost of implementing a safety measure is a key criterion. Hence, the cost of each measure is included in the models to enable costeffectiveness and cost-benefit analysis (e.g. Levin and McEwan, 2001; Nas, 1996). The most cost-effective measure is the one that meets the required effect/performance at the lowest cost. In a complete cost-benefit analysis both costs and benefits are expressed in monetary units and if the benefits exceed the costs the measure is beneficial.

In addition to the criteria presented above, several others related to, for example, consumer trust, environmental effects and the time it takes to implement safety measures can be included in the MCDA models. In general, the models include a set of criteria $(\mathrm{m})$ used to evaluating and comparing safety measures.

\subsubsection{MCDA score}

To facilitate a comparison of analysed safety measures an overall weighted preference score is calculated based on each measure's performance for the criteria. The overall preference score of a measure $\left(s_{j}\right)$ is a weighted sum calculated as

$$
s_{j}=\sum_{m} s_{j m} q_{m}
$$

where $s_{j m}$ is the preference score for measure $j$ for criterion $m$ and $q_{m} \geq 0$ represent the weights for the criteria and sum to 1 . A measure is assigned scores based on its performance for each criterion. For example, the benefit calculated using Equations (6) or (8) determines the measures' performance for the risk reduction criterion. The scores $\left(s_{j m}\right)$ are calculated by normalising the calculated performances for each criterion. The score may thus attain a value between 0 and 1 , where 1 represents the best performance. To enable the calculation of $s_{j}$, all criteria need to be mutually preference independent, i.e. preference scores assigned to all 
measures for one criterion are unaffected by the preference scores for the other criteria (Keeney and Raiffa, 1993).

For both models presented here, a compensatory or a non-compensatory evaluation strategy can be applied (Communities and Local Government, 2009). What approach is used depends on if strong performance for one criterion may compensate for weak performance for one or several other criteria. Hence, the decision-makers have to decide what strategy to be used. If a non-compensatory strategy is used, critical performance levels are defined for one or several criteria and measures not meeting the critical levels are disqualified.

The uncertainties of the MCDA results are calculated using Monte Carlo simulations to provide additional decision support (e.g. Bedford and Cooke, 2001). This makes it possible to calculate, for example, the probability of each measure having the highest score. The probabilistic approach also enables an uncertainty analysis, where the major contributions to the overall uncertainty of the analysis can be identified. This forms a basis for prioritising further collection of information to get a more reliable prioritisation of safety measures.

\subsubsection{Performance matrix}

The results of the two MCDA models are presented in performance matrices. For each measure the following information is presented: (1) the benefit of risk reduction; (2) the cost; (3) the overall preference score; (4) initial and final risk levels for the target event; and (5) the probability of not reaching an acceptable risk level for the target event (one probability for each risk type). Information on initial and final risk levels is included since it may not be necessary to reduce all risks to an acceptable level. An often used approach in risk evaluation is the ALARP (As Low As Reasonably Practicably) principle (e.g. Melchers, 2001). According to this approach a risk may be acceptable, i.e. can be left without further action, unacceptable, i.e. must be reduced, or fall within the ALARP region (see e.g. Figure 4). A risk within the ALARP region may be accepted if it is economically and/or technically unreasonable to reduce it.

\section{MODEL APPLICATIONS}

\subsection{Case study site}

The MCDA models were applied to analyse possible safety measures for the drinking water system in Bergen, Norway. The system supplies approximately 250,000 consumers and includes five surface water sources (lakes) and the same number of treatment plants. In 2004 a waterborne Giardia outbreak occurred in Bergen and up to 6,000 persons were infected. After the outbreak an external evaluation committee recommended that a risk analysis should be performed of the system from source to tap. A risk ranking with risk matrices were performed (Røstum et al., 2009; Røstum and Eikebrokk, 2008) and the results were used as input for the MCDA model applications presented here.

\subsection{Risk ranking and MCDA}

The risk ranking were based on a modified version of the Norwegian guidelines for risk and vulnerability analysis of water supply systems (NFSA, 2006) and included elements of the WSP approach and the principles of Hazard Analysis and Critical Control Points (e.g. Havelaar, 1994). Three risk types $\left(R_{k}\right)$ were included, related to: (1) water quality, i.e. health risks; (2) water quantity, i.e. supply interruptions; and (3) loss of reputation/economy. The latter risk type was used to reflect the overall effect possible events may have on the water utility organisation. For each undesired event one probability and three consequences were 
estimated using scales divided into four classes (low, medium, high and very high). Each class was defined in detail with respect to the actual probability or consequence it corresponds to. The results were presented in three risk matrices, one for each risk type. In the matrices the areas representing acceptable risk, unacceptable risk and the ALARP region were defined according to Figure 4. All three risk matrices were defined in the same way.

As part of the decision analysis the probability and consequence classes were assigned numerical values and risk priority numbers were calculated. The probability and consequence values were calculated using Equation (2) with factor $A=2$. Hence, possible values were 1, 2, 4 or 8 . The risk was calculated using Equation (3) with weights $a=b=1$ (Figure 4). The factor $(A)$ and the weights ( $a$ and $b$ ) were selected so that the final risk priority numbers corresponded the risk levels (acceptable, ALARP and unacceptable) defined in the previously performed risk ranking.

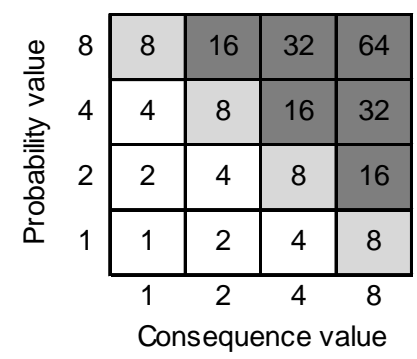

Figure 4 Risk matrix with probability and consequence values and resulting risk values. The white, light grey and dark grey represent acceptable risk (1-4), the ALARP region (8) and unacceptable risk (16-64) respectively.

In total, 85 undesired events were identified and assessed. Four events that were unacceptable with respect to at least one risk type were selected and used as target events: (1) intrusion of contaminants in the distribution system during periods of low or no water pressure, causing unacceptable water quality; (2) pipe break in the water mains due to wear or external forces, causing water quantity and quality problems; (3) failure of UV disinfection due to power failure, causing water quality problems; and (4) raw water scarcity due to a long drought, causing water quantity problems.

Possible safety measures were identified for the four target events, see Table 2. Some measures affect only the target event but others affect one or two other events that do not have to be target events. For all affected events the expected probability and consequence values representing the situation after the measures are implemented were estimated. For the discrete model, the distributions in Table 1 were used when estimating probability and consequence values. These distributions were considered as a reasonable starting point representing a relatively high uncertainty. For the beta model, the probabilities of achieving acceptable risk levels were modelled using the Beta distributions in Table 3. All distributions in Table 3 have the same level of uncertainty, $\alpha+\beta=42$, and were considered a reasonable starting point. Alternative sets of uncertainty distributions (Figures 2 and 3 ) were not used in this example. For both models the Monte Carlo simulations were performed using 10,000 iterations. 
Table 2 Possible safety measures for the four target events. For each measure it is specified what target event it is designed for and the number of additional events, which do not have to be a target events, the measure affects.

\begin{tabular}{clcc}
\hline Ref. & Measure & $\begin{array}{c}\text { Target } \\
\text { event no. }\end{array}$ & $\begin{array}{c}\text { No. of additional } \\
\text { events affected }\end{array}$ \\
\hline 1.1 & Repair under pressure & 1 & 0 \\
1.2 & Increase rehabilitation rate in the distribution network & 1 & 2 \\
1.3 & Replace valves & 1 & 0 \\
1.4 & New critical control point & 1 & 0 \\
1.5 & More frequent recommendations for boiling & 1 & 0 \\
\hline 2.1 & New pipeline & 2 & 2 \\
\hline 3.1 & Monitor power supply & 3 & 0 \\
3.2 & Increase UV capacity & 3 & 0 \\
3.3 & Additional treatment barrier & 3 & 1 \\
3.4 & Install system for uninterrupted power supply & 3 & 1 \\
3.5 & Install emergency power supply & 3 & 1 \\
\hline 4.1 & New reservoir & 4 & 0 \\
4.2 & Repair leaks & 4 & 1 \\
4.3 & Reduce water use & 4 & 0 \\
4.4 & New raw water intake & 4 & 0 \\
\hline
\end{tabular}

Table 3 Beta distributions for the probability of achieving an acceptable risk level. For all distributions $\alpha+\beta=42$.

\begin{tabular}{cccccc}
\hline \multirow{2}{*}{$\begin{array}{c}\text { Risk priority } \\
\text { number }\end{array}$} & \multicolumn{3}{l}{ Probability of acceptable risk } & \multirow{2}{*}{$\beta$} & \multirow{2}{*}{$\beta$} \\
\cline { 2 - 5 } & Most likely & P05 & P95 & & $\beta$ \\
\hline 64 & 0.00 & 0.00 & 0.07 & 1 & 41 \\
\hline 32 & 0.10 & 0.05 & 0.21 & 5 & 37 \\
\hline 16 & 0.30 & 0.20 & 0.43 & 13 & 29 \\
\hline 8 & 0.50 & 0.37 & 0.63 & 21 & 21 \\
\hline 4 & 0.70 & 0.57 & 0.80 & 29 & 13 \\
\hline 2 & 0.90 & 0.79 & 0.95 & 37 & 5 \\
\hline 1 & 1.00 & 0.93 & 1.00 & 41 & 1 \\
\hline
\end{tabular}

The benefit of risk reduction was calculated based on the assumption that the three risk types $\left(R_{k}, k=1,2,3\right)$ were equally important. The criteria on risk reduction, including the three risk types, and cost were also considered equally important. The reason for considering the risk types and the two criteria equally important was to facilitate model evaluation and keep the results as transparent as possible.

The costs for implementing the measures were estimated qualitatively as low, low/medium, medium, medium/high or high. This kind of qualitative estimates may cause limitations since it becomes harder to calculate, for example, benefit to cost ratios and use other optimisation models. If possible, estimates of the costs in monetary units should thus be used to provide more detailed information. It was, however, not possible quantify the costs in monetary units in this application and this may be the situation also in other applications. In both models, discrete probability distributions were used to model uncertainties about the true cost categories (Table 4). The distributions in Table 4 all have a normalised entropy value of 0.63 . The relative difference between the cost categories high, medium/high, medium, medium/low and low were considered to be equal and they were therefore translated into preference scores $0,0.25,0.50,0.75$ and 1 respectively. By normalising the calculated benefits of risk reduction, i.e. dividing with the highest value, they were translated into preference scores with the highest value of 1 . 
Discrete probability distributions used to model uncertainties in the costs. All distributions have a normalised entropy value of 0.63 .

\begin{tabular}{cccccc}
\hline & \multicolumn{5}{c}{ Probability of each cost category } \\
\cline { 2 - 6 } Cost & Low & Low/medium & Medium & Medium/high & High \\
\hline Low & 0.68 & 0.16 & 0.09 & 0.04 & 0.03 \\
\hline Low/medium & 0.10 & 0.70 & 0.10 & 0.06 & 0.04 \\
\hline Medium & 0.05 & 0.10 & 0.70 & 0.10 & 0.05 \\
\hline Medium/high & 0.04 & 0.06 & 0.10 & 0.70 & 0.10 \\
\hline High & 0.03 & 0.04 & 0.09 & 0.16 & 0.68 \\
\hline
\end{tabular}

\section{RESULTS}

The performance matrices, Tables 5 and 6, summarise the case study results for the discrete and the beta models. According to the discrete model, measure 1.2 (increased rehabilitation rate) clearly has the highest benefit of the measures for target event 1 (contaminant intrusion), see Table 5. One reason is that the measure affects three events (Table 2). However, measure 1.2 has a medium/high cost and the preference score is highest for measure 1.1 (repair under pressure), which has a lower benefit but also a lower cost. For UV disinfection failure (target event 3), measure 3.1 (monitor power supply) has the lowest benefit but also the lowest cost and therefore the highest score (Table 5). In the discrete model the measures with the highest score for target events 1, 3 and 4 do not reduce all risks to an acceptable level (Table 5). This situation may arise if not critical levels of performance are defined and thus strong performance for one criterion is allowed to compensate for weak performance for other criteria. A non-compensatory approach can be applied by, for example, disqualifying measures that do not reduce all risks to an acceptable level for the target event.

In the beta model the final risk level, or more precisely the probability of achieving an acceptable risk, is included when the benefit is calculated. In the performance matrix (Table 6 ) it can be seen that the measure with highest score for each target event also reduce the risks to an acceptable level. A comparison of the scores from the two models (Figure 5) shows that measures reducing all target event risks to an acceptable level are ranked higher in the beta model compared to the discrete model (measures 1.2, 2.1, 3.4, 3.5, 4.1 and 4.4).

The initial and final risk levels are included in the performance matrices to show whether the risks are acceptable, within the ALARP region or unacceptable. For target event 1 we can in Table 6 (the beta model) see that measure 1.2 has the highest score, reduces all risks to an acceptable level but is also associated with the highest costs. Safety measures 1.1 and 1.4 (new critical control point) both have the second highest preference score, reduce the quality risk to the ALARP region and are associated with the lowest cost. If it is not reasonable due to technical and/or economic reasons to select measure 1.2, then measure 1.1 or 1.4 could be selected based on the ALARP principle.

The diamonds, triangles and circles in the performance matrices show the most likely risk levels (i.e. unacceptable, ALARP or acceptable) according to the definition in Figure 4. The probability of not having an acceptable risk gives information about the uncertainty in the final risk level. Hence, measures resulting in the same final risk levels can be compared using this information (e.g. the quantity risk for measures 4.1 and 4.4 in Table 6). 
Table $5 \quad$ Performance matrix for the discrete model including benefits $\left(u_{j}\right)$, costs and preference scores $\left(s_{j}\right)$ for each measure $(j)$. For the target events $(t)$ the initial risk levels $\left(R_{0 t k}\right)$ are presented as well as the final risk levels $\left(R_{j t k}\right)$ after measure $j$ has been implemented. Also the probability of the final risk being higher than the acceptable risk $\left(R_{k}^{c}\right)$ are presented. Diamonds represent unacceptable risks, triangles represent risks within the ALARP region and circles represent acceptable risks.

\begin{tabular}{|c|c|c|c|c|c|c|c|c|c|}
\hline \multirow{2}{*}{$\begin{array}{c}\text { Mcasure } \\
(j)\end{array}$} & \multirow[b]{2}{*}{$u_{j}$} & \multirow[b]{2}{*}{$\operatorname{Cos} 1$} & \multirow[b]{2}{*}{$s_{j}$} & \multicolumn{3}{|c|}{$R_{0 t k} \rightarrow R_{j t k}$} & \multicolumn{3}{|c|}{$P\left(R_{j t k}>R_{k}^{c}\right)$} \\
\hline & & & & qual. & quan. & rep. & qual. & quan. & rep. \\
\hline 1.1 & 13.3 & Low & 0.50 & $\rightarrow$ & $\bigcirc \rightarrow \bigcirc$ & $\Delta \rightarrow$ & 0.75 & 0.19 & 0.30 \\
\hline 1.2 & 69.8 & Mediamhigh & 0.47 & $\rightarrow$ & $\bigcirc \rightarrow \bigcirc$ & $\Delta \rightarrow 0$ & 0.30 & 0.19 & 0.19 \\
\hline 1.3 & 13.1 & Medium & 0.32 & $\rightarrow$ & $\rightarrow \bigcirc$ & $\Delta \rightarrow \bigcirc$ & 0.76 & 0.19 & 0.30 \\
\hline 1.4 & 13.0 & Low & 0.49 & $\rightarrow \triangle$ & $\rightarrow \bigcirc$ & $\Delta \rightarrow 0$ & 0.77 & 0.19 & 0.30 \\
\hline 1.5 & 8.9 & Medium & 0.29 & $\rightarrow$ & $\rightarrow 0$ & $\Delta \rightarrow$ & 0.20 & 0.20 & 0.86 \\
\hline 2.1 & 51.9 & Medium & 0.49 & $\rightarrow \bigcirc$ & $>\rightarrow 0$ & $\Delta \rightarrow 0$ & 0.11 & 0.12 & 0.37 \\
\hline 3.1 & 9.9 & Iow & 0.48 & & $\Delta \rightarrow \triangle$ & $\rightarrow$ & 0.86 & 0.75 & 0.87 \\
\hline 3.2 & 10.8 & Medium & 0.30 & & $\Delta \rightarrow \Delta$ & & 0.87 & 0.75 & 0.86 \\
\hline 3.3 & 17.2 & Iligh & 0.15 & & $\Delta \rightarrow$ & & 0.76 & 0.76 & 0.88 \\
\hline 3.4 & 41.3 & Medium & 0.45 & & $\Delta \rightarrow 0$ & $\rightarrow$ & 0.19 & 0.20 & 0.30 \\
\hline 3.5 & 41.3 & Medium & 0.45 & $\rightarrow C$ & $\Delta \rightarrow 0$ & $>\rightarrow 0$ & 0.20 & 0.19 & 0.29 \\
\hline 4.1 & 31.2 & Mcdium/high & 0.31 & $\rightarrow C$ & $\rightarrow$ & $\Delta \rightarrow 0$ & 0.13 & 0.20 & 0.20 \\
\hline 4.2 & 42.9 & Medium/high & 0.36 & $\rightarrow C$ & & $\Delta \rightarrow 0$ & 0.20 & 0.87 & 0.30 \\
\hline 4.3 & 17.0 & Medium & 0.34 & & & $\Delta \rightarrow \bigcirc$ & 0.19 & 0.87 & 0.30 \\
\hline 4.4 & 33.0 & High & 0.24 & $\rightarrow 0$ & $>\rightarrow 0$ & $\Delta \rightarrow 0$ & 0.12 & 0.13 & 0.19 \\
\hline
\end{tabular}

Table $6 \quad$ Performance matrix for the beta model including benefits $\left(u_{j}\right)$, costs and preference scores $\left(s_{j}\right)$ for each measure $(j)$. For the target events $(t)$ the initial risk levels $\left(R_{0 t k}\right)$ are presented as well as the final risk levels $\left(R_{j t k}\right)$ after measure $j$ has been implemented. Also the probability of the final risk being higher than the acceptable risk $\left(R_{k}^{c}\right)$ are presented. Diamonds represent unacceptable risks, triangles represent risks within the ALARP region and circles represent acceptable risks.

\begin{tabular}{|c|c|c|c|c|c|c|c|c|c|}
\hline \multirow{2}{*}{$\begin{array}{c}\text { Measure } \\
(j)\end{array}$} & \multirow[b]{2}{*}{$u_{j}$} & \multirow[b]{2}{*}{ Cost } & \multirow[b]{2}{*}{$s_{j}$} & \multicolumn{3}{|c|}{$R_{0 t k} \rightarrow R_{j t k}$} & \multicolumn{3}{|c|}{$P\left(R_{j t k}>R_{k}^{c}\right)$} \\
\hline & & & & qual. & quan. & rep. & qual. & quan. & rep. \\
\hline 1.1 & 8.5 & Iow & 0.50 & $\rightarrow$ & $\rightarrow \rightarrow$ & $\Delta \rightarrow 0$ & 0.50 & 0.12 & 0.31 \\
\hline 1.2 & 57.7 & Medium/high & 0.65 & $\rightarrow$ & $\rightarrow$ & $\Delta \rightarrow 0$ & 0.31 & 0.12 & 0.12 \\
\hline 1.3 & 8.5 & Medium & 0.32 & $\rightarrow$ & $\rightarrow$ & $\Delta \rightarrow \bigcirc$ & 0.50 & 0.12 & 0.31 \\
\hline 1.4 & 8.5 & Low & 0.50 & $\rightarrow$ & $\rightarrow 0$ & $\Delta \rightarrow \bigcirc$ & 0.50 & 0.12 & 0.31 \\
\hline 1.5 & 8.6 & Medium & 0.32 & & $0 \rightarrow 0$ & $\Delta \rightarrow$ & 0.12 & 0.12 & 0.69 \\
\hline 2.1 & 53.6 & Medium & 0.72 & $\rightarrow$ & $>\rightarrow 0$ & $\Delta \rightarrow \bigcirc$ & 0.02 & 0.02 & 0.31 \\
\hline 3.1 & 5.0 & Iow & 0.47 & & $\rightarrow$ & & 0.69 & 0.50 & 0.69 \\
\hline 3.2 & 5.0 & Medium & 0.29 & & $\rightarrow \triangle$ & & 0.69 & 0.50 & 0.69 \\
\hline 3.3 & 14.8 & Iligh & 0.20 & & $\rightarrow \triangle$ & & 0.50 & 0.50 & 0.69 \\
\hline 3.4 & 42.8 & Medium & 0.62 & & $\Delta \rightarrow 0$ & & 0.12 & 0.12 & 0.31 \\
\hline 3.5 & 42.7 & Medium & 0.62 & 2 & $\Delta \rightarrow 0$ & 1 & 0.12 & 0.12 & 0.31 \\
\hline 4.1 & 34.6 & Medium/high & 0.46 & $\rightarrow C$ & & $\Delta \rightarrow O$ & 0.02 & 0.12 & 0.12 \\
\hline 4.2 & 28.2 & Medium/high & 0.40 & $\rightarrow$ & & $\Delta \rightarrow \bigcirc$ & 0.12 & 0.69 & 0.31 \\
\hline 4.3 & 9.5 & Medium & 0.33 & $\rightarrow$ & & $\Delta \rightarrow 0$ & 0.12 & 0.69 & 0.31 \\
\hline 4.4 & 38.5 & High & 0.40 & $P \rightarrow 0$ & $>\rightarrow 0$ & $\Delta \rightarrow 0$ & 0.02 & 0.02 & 0.12 \\
\hline
\end{tabular}

Using information on final risk levels and costs, the measures can be analysed from costeffectiveness and cost-benefit perspectives. If it is required that all risks must be reduced to an acceptable level, then the measure that fulfil this criterion at the lowest cost is most costeffective. For UV disinfection failure (target event 3), measures 3.4 and 3.5 (uninterrupted 
power supply and emergency power supply) both fulfil the criterion to the same cost and are therefore equally cost-effective (Tables 5 and 6). For raw water scarcity (target event 4), measures 4.1 and 4.4 (new reservoir and new raw water intake) both fulfil the criterion but 4.1 does it at the lowest cost and is thus most cost-effective.

If the measures are analysed from a cost-benefit perspective one is interested to see if the benefits exceed the costs. Since neither the benefits nor the costs are expressed in monetary units this analysis can only be done qualitatively here. A high benefit to a low cost is of course preferred over a low benefit to a high cost. If both the benefit and cost is high or low, it is more uncertain whether or not the measure results in a positive net benefit. From a costbenefit approach one could argue whether measures 1.1 with a relatively low benefit and a low cost has a higher net benefit compared to measure 1.2 with a higher benefit but also to a higher cost.

For target event 2 (pipe break in the water mains), only one measure was analysed (new water main) and it can therefore only be compared to measures for other target events. The results from the MCDA models should first of all be used to evaluate and compare measures designed for the same target event. Since all target events included in this case study were associated with at least one unacceptable risk, they all need to be reduced. However, since resources for risk reduction are limited it may be necessary to also prioritise which measures to implement first. This prioritisation can be based on what initial risk levels are considered to be most severe, or by comparing the preference score of the selected measures.

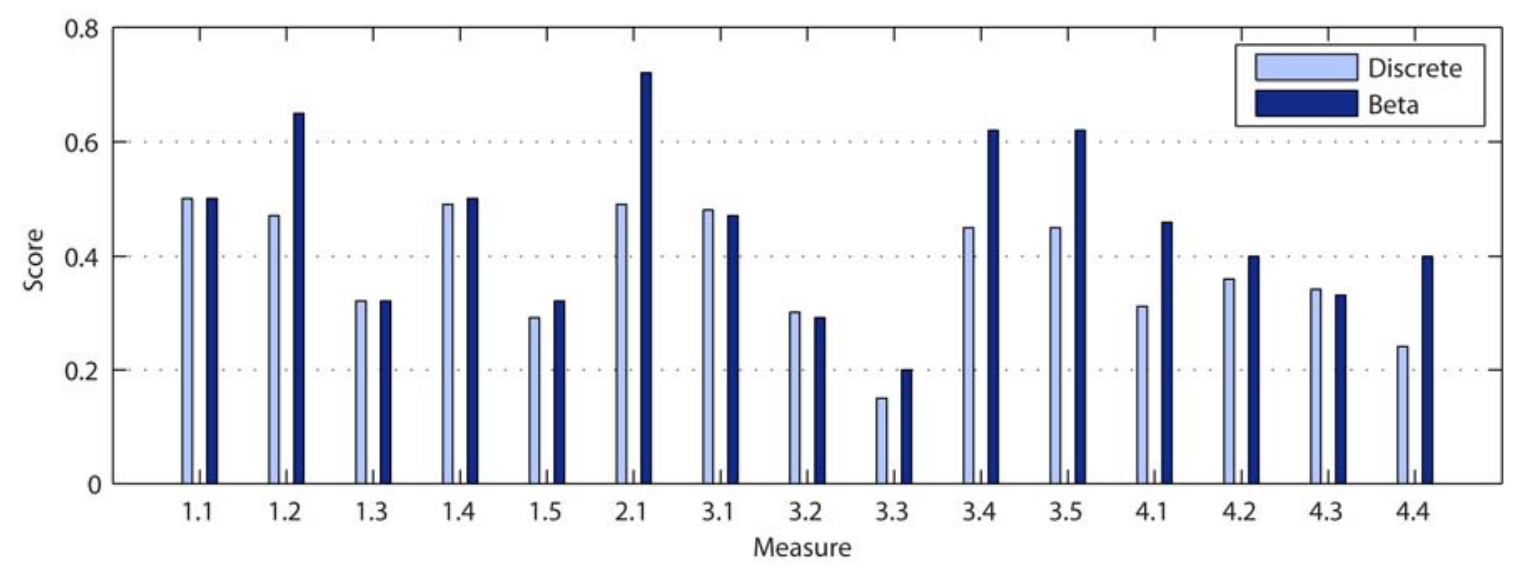

Figure 5 Preference scores $\left(s_{j}\right)$ from the two MCDA models.

The scores presented in the performance matrices are expected values. Based on the uncertainties of the preference scores the probability of each measure having the highest score can be calculated. The histograms in Figure 6 show these probabilities for target events 1, 3 and 4 . The histograms illustrate the difference between the scores with consideration to uncertainties. For example, for target event 1 the probability of measures 1.1, 1.2 and 1.4 are fairly similar in the discrete model, whereas measure 1.2 has clearly the highest score in the beta model. It should be stressed that the histograms in Figure 6 include safety measures that result in unacceptable risk levels. If only measures that reduce the risks to an acceptable or ALARP level are included in the calculations, the results presented in Figure 7 are obtained. For target event 1 only measure 1.5 is disqualified and the results in Figure 7 are similar to the ones in Figure 6. For target events 3 and 4, the two models give similar results (Figure 7) where measures 3.4 and 3.5 have probabilities close to 0.5 and measure 4.1 has a higher probability than measure 4.4 . 

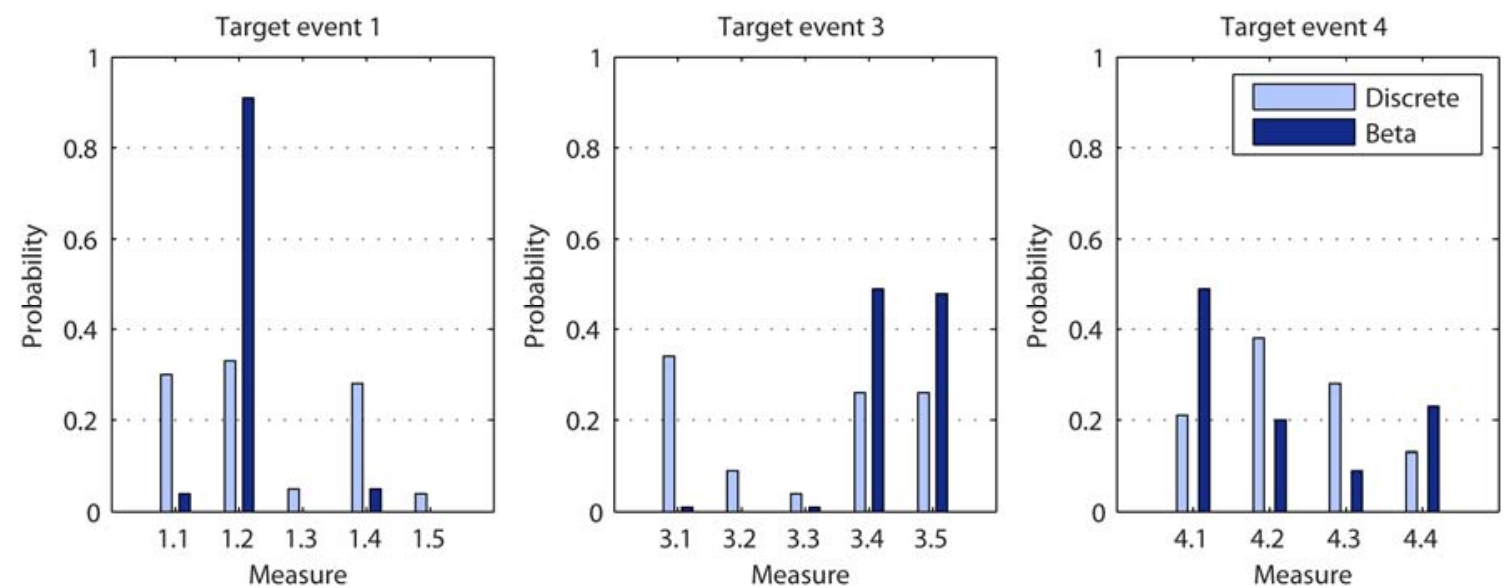

Figure 6 Histograms showing the probability of each measure having the highest preference score for target events 1, 3 and 4 (contaminant intrusion, UV disinfection failure and raw water scarcity respectively). Note that all measures are included, also those resulting in unacceptable risk levels.
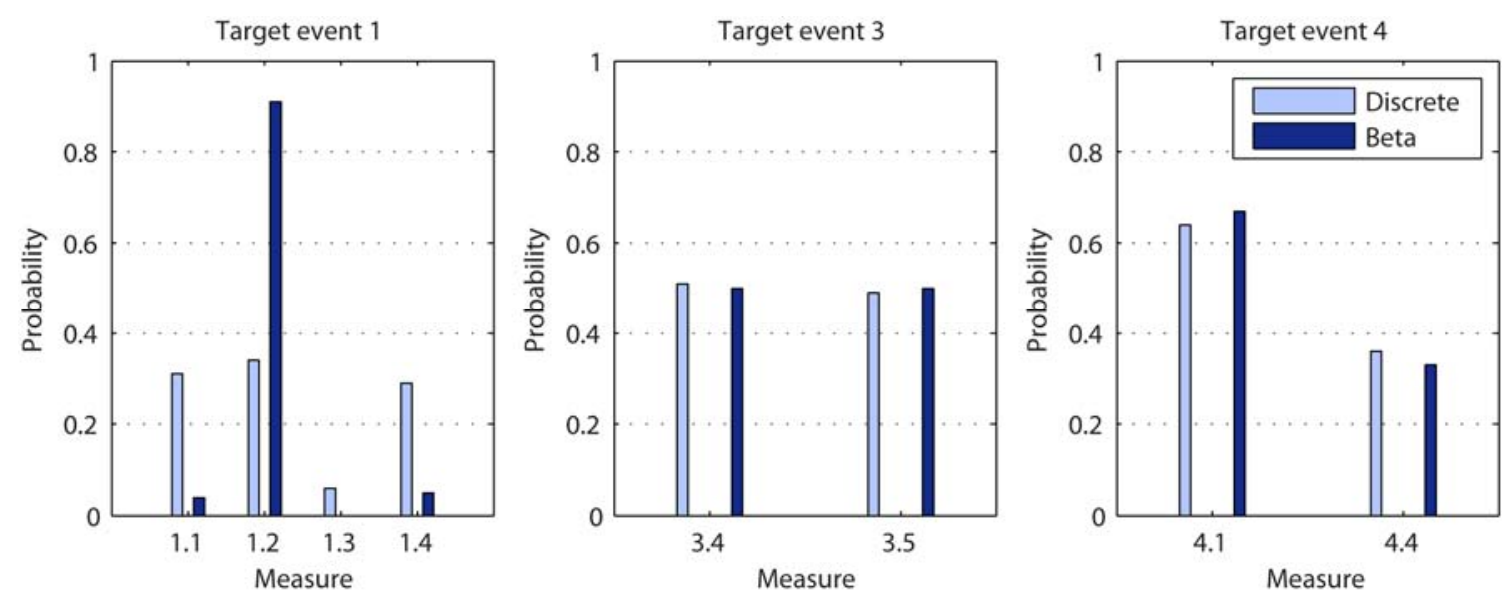

Figure 7 Histograms showing the probability of each measure having the highest overall preference score for target events 1, 3 and 4 (contaminant intrusion, $U V$ disinfection failure and raw water scarcity respectively). All measures resulting in unacceptable risk levels are excluded.

If, for the discrete model, all measures not obtaining acceptable risk levels are disqualified, then the two models give the highest score to the same measures in the performed case study. If this approach is used, then the risk related to intrusion of contaminants in the distribution network (target event 1) should be reduced by an increased rehabilitation rate (1.2). The risk of UV disinfection failure (target event 3 ) should be reduced by installing a system for uninterrupted power supply (3.4) or an emergency power supply (3.5), both are ranked equally. The fourth target event, raw water scarcity, should be treated by installing a new reservoir (4.1). For target event 2, pipe break in the water mains, a new pipeline is the only suggested measure (2.1). These measures $(1.2,2.1,3.4,3.5$ and 4.1) are also the ones that should be selected if a cost-effectiveness approach is used and it is required that the risks must be reduced to an acceptable levels. It should be noted that these results are based on the classification, assumptions etc. made in this analysis. The final prioritisation may be different if it is considered reasonable to leave some risks within the ALARP region or if a cost-benefit approach is used. 


\section{DISCUSSION}

The two MCDA models differ in the way uncertainties are assessed and how the benefit of risk reduction is calculated. Hence, whether the discrete or beta model should be used depends mainly on: (1) what is considered to be the best way of handling uncertainties; and (2) how the decision-maker perceives the benefit of risk reduction. Due to different ways of calculating the benefit, measures reducing all risk to an acceptable level get a higher relative score in the beta model compared to the discrete model. In both models the risk levels presented in the performance matrices and the probability of the final risk level not being acceptable can be used to disqualify measures not meeting predefined performance levels. It is important to consider the ALARP region since the acceptance criteria in the matrices are not free of uncertainties, and it may be acceptable to have risks within the ALARP region if is unreasonable to reduce them due to technical and/or economic reasons.

The results of the two models allows for different types of evaluation and comparison. The measures can, for example, be compared based on the preference score, critical performance levels can be defined and the cost-effectiveness as well as cost-benefit aspects can be considered. A compensatory or non-compensatory approach can also be applied and the choice affects the results. What approach to use has to be decided by the decision-maker. Furthermore, the weights reflecting the importance of the different risk types and criteria need to be evaluated and defined to reflect the decision-makers' view of the decision problem. In addition to the criteria

The suggested MCDA models make it possible to analyse uncertainties in different ways. In the result section the probability of not achieving an acceptable risk level and the probability of each measure having the highest preference score are presented. These results were calculated based on the uncertainties in input data and should be regarded as relative values. Hence, these results can be compared within the analysis but cannot be compared to results from other analyses. Although not done in the case study presented here, it is reasonable to analyse how changes in model parameters such as the weights affects the prioritisation of safety measures. Furthermore, by calculating rank correlation coefficients it is possible to identify what input data that contribute most to the uncertainties in the results. This information can be used to identify where additional information should be collected to most effectively reduce the uncertainties in the results.

A set of probability distributions will most likely facilitate applications of the models (Figures 2 and 3). To define distributions in a consistent manner the sum of the shape parameters $(\alpha+\beta)$ can be used as a measure of uncertainty for the Beta distributions. For the discrete distribution the normalised entropy can be used.

The MCDA results can be used to select which safety measure is most suitable for each target event but also to prioritise which measure to implement first. It may be relevant to compare combinations of measures and in such cases new measures including all effects and costs should be defined and included in the analysis. The interaction between different safety measures has to be considered since measures may, for example, affect the same events and some measures may not be possible to combine. It is therefore not possible to simply add together the risk reduction of separate measures.

The presented models provide a stepwise procedure that makes it possible to use risk ranking to not only prioritise risks but also evaluate and compare possible safety measures and consider uncertainties in a structured manner. The need of the developed models was identified based on problems related to the drinking water sector but the need of more formal handling of risk and uncertainty in MCDA is also emphasised in the literature. The presented models provide approaches and techniques generally applicable within the field of MCDA 
and the outcomes include: (1) a stepwise procedure for prioritising safety measures; (2) two approaches (beta and discrete) for modelling uncertainties; and (3) two techniques (the sum of the shape parameters and the normalised entropy) for defining probability distributions with the same degree of uncertainty.

The case study shows specifically how MCDA-based risk analysis techniques can be applied within the drinking water sector. The models constitute tools that make it possible for water utilities to assess risks to the entire drinking water system and prioritise water safety measures so that available resources are used efficiently. This contributes to an efficient risk management according to the WSP approach emphasised by the WHO.

Calculations for the MCDA models are easily performed using standard spreadsheet software and an add-in software for running Monte Carlo simulations. The model descriptions and applications presented in this paper show how to combine risk ranking and MCDA but the basics of the models can be combined with any risk assessment as long as the risk-reduction from possible measures can be estimated. Lindhe et al. (2011) present a similar approach where a quantitative risk assessment method is combined with economic analysis and results similar to the ones from the MCDA models are obtained.

\section{CONCLUSIONS}

The main conclusions of this study are:

- Evaluation of water safety measures may include several aspects which can be hard to analyse in a structured manner. The models described here provide a stepwise approach that makes it possible to evaluate and compare safety measures in a transparent manner.

- Risk ranking can successfully be combined with MCDA. The presented MCDA models provide two possible approaches for evaluating safety measures. Which model should be used mainly depends on how one wants to include uncertainties and what way of calculating the benefit of risk reduction is preferred. Both models can be adjusted by assigning different weights to the included criteria.

- The results of the MCDA models can be evaluated using different approaches and it is up to the decision-maker to select what approach is most suitable. Preference scores should be analysed in combination with information on risk levels in order to avoid, for example, too small risk reduction.

- By including uncertainties in the MCDA models the risk levels are not assumed to be free of uncertainty, which is typically the case in risk ranking methods.

- The suggested models provide approaches and techniques for evaluating safety measures and modelling uncertainties in MCDA that are not dependent on the application domain. Furthermore, the case study relates the models to a real world problem and shows how MCDA-based risk analysis techniques can be applied within the drinking water sector.

- It should be stressed that MCDA models do not make decisions but provide decision support based on the information and aspects included.

\section{ACKNOWLEDGEMENTS}

This study has been carried out within the framework of the TECHENAU project, funded by the European Commission under the $6^{\text {th }}$ framework programme (www.techneau.org), and with support from the Swedish Water \& Wastewater Association. 


\section{REFERENCES}

AZ/NZS. Handbook: Risk Management Guidelines - Companion to AS/NZS 4360:2004. Standards Australia/Standards New Zealand, 2004.

Bartram J, Corrales L, Davison A, Deere D, Drury D, Gordon B, et al. Water safety plan manual: step-by-step risk management for drinking-water suppliers. Geneva: World Health Organization, 2009.

Bedford T, Cooke RM. Probabilistic risk analysis: foundations and methods. Cambridge: Cambridge University Press, 2001.

Bouchard C, Abi-Zeid I, Beauchamp N, Lamontagne L, Desrosiers J, Rodriguez M. Multicriteria decision analysis for the selection of a small drinking water treatment system. Journal of Water Supply: Research and Technology-AQUA 2010; 59: 230242.

Box GEP, Hunter JS, Hunter WG. Statistics for Experimenters: Design, Innovation and Discovery. Hoboken, N.J.: Wiley-Interscience, 2005.

Burgman MA. Risks and decisions for conservation and environmental management. Cambridge: Cambridge University Press, 2005.

Communities and Local Government. Multi-criteria analysis: a manual. Department for Communities and Local Government, 2009.

Cox AL. What's Wrong with Risk Matrices? Risk Analysis 2008; 28: 497-512.

Davison A, Howard G, Stevens M, Callan P, Fewtrell L, Deere D, et al. Water Safety Plans: Managing drinking-water quality from catchment to consumer. World Health Organization, Geneva, 2005.

Hajkowicz S, Collins K. A Review of Multiple Criteria Analysis for Water Resource Planning and Management. Water Resources Management 2007; 21: 1553-1566.

Havelaar AH. Application of HACCP to drinking water supply. Food Control 1994; 5: 145152.

Hyde KM, Maier HR, Colby CB. Reliability-Based Approach to Multicriteria Decision Analysis for Water Resources. Journal of Water Resources Planning and Management 2004; 130: 429-438.

IEC. Dependability Management - Part 3: Application guide - Section 9: Risk analysis of technological systems. International Electrotechnical Commission, 1995.

Jaynes ET. Prior Probabilities. Systems Science and Cybernetics, IEEE Transactions on 1968; 4: $227-241$.

Joerin F, Cool G, Rodriguez MJ, Gignac M, Bouchard C. Using multi-criteria decision analysis to assess the vulnerability of drinking water utilities. Environmental Monitoring and Assessment 2009; 166: 313-30.

Kaplan S, Garrick BJ. On The Quantitative Definition of Risk. Risk Analysis 1981; 1: 11-27. 
Kaplan S, Haimes YY, Garrick BJ. Fitting hierarchical holographic modeling into the theory of scenario structuring and a resulting refinement to the quantitative definition of risk. Risk Analysis 2001; 21: 807-819.

Keeney RL, Raiffa H. Decision with Multiple Objectives: Preference and Value Tradeoffs. Cambridge: Cambridge University Press, 1993.

Levin HM, McEwan PJ. Cost-effectiveness analysis: Methods and applications. Thousand Oaks, California: Sage Publications, 2001.

Lindhe A, Rosén L, Norberg T, Bergstedt O. Fault tree analysis for integrated and probabilistic risk analysis of drinking water systems. Water Research 2009; 43: 16411653.

Lindhe A, Rosén L, Norberg T, Bergstedt O, Pettersson TJR. Cost-effectiveness analysis of risk-reduction measures to reach water safety targets. Water Research 2011; 45: 241253.

Melchers RE. On the ALARP approach to risk management. Reliability Engineering \& System Safety 2001; 71: 201-208.

Nas TF. Cost-benefit analysis: Theory and application. Thousand Oaks, Calif:: Sage Publications, 1996.

NFSA. Improved safety and emergency preparedness in water supply: Guidance (In Norwegian). Norwegian Food Safety Authority Oslo, 2006.

Rosén L, Gustafson G. A Bayesian Markov Geostatistical Model for Estimating Hydrogeological Properties. Ground Water 1996; 34: 865-875.

Rosén L, Lindhe A, Bergstedt O, Norberg T, Pettersson TJR. Comparing risk-reduction measures to reach water safety targets using an integrated fault tree model. Water Science and Technology: Water Supply 2010; 10: 428-436.

Rosenbaum MS, Rosén L, Gustafson G. Probabilistic Models for Estimating Lithology. Engineering Geology 1997; 47: 43-55.

Roy B. Paradigms and Challenges. In: Figueira J, Greco S, Ehrogott M, editors. Multiple Criteria Decision Analysis: State of the Art Surveys. 78. Springer, New York, 2005, pp. 3-24.

Røstum J, Aasen A, Eikebrokk B. Risk and Vulnerability Assessment ("Ros-Analysis") of the Bergen Water Supply System - A Source to Tap Approach. In: Hlavinek P, Popovska C, Marsalek J, Mahrikova I, Kukharchyk T, editors. Risk Management of Water Supply and Sanitation Systems. Springer, 2009, pp. 73-83.

Røstum J, Eikebrokk B. Risk and vulnerability analysis of the Bergen water supply system (In Norwegian). SINTEF, 2008.

Stewart T. Dealing with Uncertainties in MCDA. In: Figueira J, Greco S, Ehrogott M, editors. Multiple Criteria Decision Analysis: State of the Art Surveys. 78. Springer, New York, 2005, pp. 445-466. 
WHO. Guidelines for drinking-water quality. $4^{\text {th }}$ edition. World Health Organization, Geneva, 2011. 